\title{
PLGA-PEG-supported Pd Nanoparticles as Efficient Catalysts for Suzuki-Miyaura Coupling Reactions in Water
}

\author{
Anaëlle Dumas $\S^{\star}$, Arnaud Peramo, Didier Desmaële, and Patrick Couvreur \\ §SCS-DSM Award for best poster presentation in Chemical Biology
}

\begin{abstract}
Chemical transformations that can be performed selectively under physiological conditions are highly desirable tools to track biomolecules and manipulate complex biological processes. Here, we report a new nanocatalyst consisting of small palladium nanoparticles stabilized on the surface of PLGA-PEG nanoparticles that show excellent catalytic activity for the modification of biological building blocks through Suzuki-Miyaura cross-coupling reactions in water. Brominated or iodinated amino acids were coupled with aryl boronic acids in phosphate buffer in good yields. Interestingly, up to $98 \%$ conversion into the coupled amino acid could be achieved in $2 \mathrm{~h}$ at $37^{\circ} \mathrm{C}$ using the stable, water-soluble cyclic triolborate as organometallic partner in the presence of only $1 \mathrm{~mol} \%$ of palladium. These results pave the way for the modification of biomolecules in complex biological systems such as the intracellular space.
\end{abstract}

Keywords: Amino acid chemistry · Palladium nanoparticles · Suzuki-Miyaura cross-coupling

\section{Introduction}

The tracking and manipulation of individual cellular components provide unprecedented opportunities to understand complex cellular processes and may open new avenues for the treatment of human disease. ${ }^{[1]}$ Among the functional tools available for the manipulation of biological systems, 'bioorthogonal reactions' offer versatile platforms for the selective and controlled modification of biomolecules of interest.[1] These transformations involve covalent bond formation between non-native chemical tags that react highly chemoselectively together under physiological conditions, while remaining inert towards the myriad of other functional groups found in biological environments. The major difficulty resides in finding non-perturbing chemical handles that react rapidly, while generating no or non-toxic side products. ${ }^{[2]}$ As a result, only a hand-

\footnotetext{
${ }^{*}$ Correspondence: Dr. A. Dumas Institut Galien Paris-Sud

UMR 8612, CNRS, Univ. Paris-Sud

Université Paris-Saclay

Faculté de Pharmacie

5 rue JB Clément

92296 Châtenay-Malabry, France

E-mail: anaelle.dumas@u-psud.fr
}

ful of reactions have been developed that fulfil all of these criteria. ${ }^{[3]}$ Among them, the Staudinger ligation (azide-phosphine), copper-catalysed or strain-promoted azide-alkyne cycloadditions and tetrazine ligation approaches are the most widely utilised bioorthogonal reactions to date. ${ }^{[4]}$ While these transformations have been helpful addressing a number of fundamental biological questions, ${ }^{[1 b, 5]}$ toxicity, cross-reactivity with endogenous thiols or other biomolecules, and poor stability of the reagents in biological environments, complicate their application in a number of settings. ${ }^{[6]}$ Therefore, the design of truly bioorthogonal reactions, involving stable, selective and synthetically accessible reaction partners remains an area of active investigation in chemical biology. ${ }^{[3,7]}$

Palladium-mediated cross-coupling reactions provide new possibilities for chemical reporter strategies. ${ }^{[8]}$ The reactive groups associated with these processes are rarely found in natural systems and cross-coupling reactions proceed with high specificity and broad functional group tolerance. However, the poor stability under aerobic conditions of most palladium $(0)$ complexes used as catalysts, and the high temperatures required for activation, have restricted these reactions to the conventional organic synthesis of small molecules. In the last decade, efforts to develop water- and air-stable catalytic systems active within the narrow biologically-relevant $\mathrm{pH}$ and temperature windows, have enabled the use of palladium reactions in bio- logical settings. ${ }^{66,9]}$ Palladium-catalysed cross-coupling reactions involving protein substrates suffered from low yields until Davis and coworkers reported the 2-amino-4,6-dihydroxypyrimidine (ADHP)based catalyst $\left[\mathrm{Pd}(\mathrm{OAc})_{2}(\mathrm{ADHP})_{2}\right]$ enabling efficient Suzuki-Miyaura reaction of halogenated amino acids and peptides with boronic acids. ${ }^{[10]}$ This system proved its efficacy in the preparation of structurally-defined protein-conjugates with excellent conversions at chemically-installed or genetically-encoded aryl iodides on purified proteins and on the surface of bacteria. ${ }^{[10,11]}$ In parallel, the analogue $N, N$ '-dimethyl ADHP-palladium(II) complex was reported by Li et al. to promote copper-free Sonogashira cross-coupling reactions on alkyne-labelled proteins inside bacterial cells. ${ }^{[12]}$

These results propelled palladium-mediated reactions in the very demanding bioorthogonal chemistry toolbox. However, these transformations still suffer from drawbacks as the non-specific interactions of the homogenous palladium complexes with nitrogen-, sulphur-, or oxygen-containing biological building blocks may cause cytotoxicity ${ }^{[12]}$ and require the removal of the palladium catalyst after reaction using metal scavengers. ${ }^{[1]}$ The use of heterogeneous palladium catalysts could overcome these problems by reducing non-specific binding to biomolecules. ${ }^{8]}$ Palladium nanoparticles (Pd NPs) stabilised by organic polymers, or inorganic supports have already demonstrated their 
activity in Suzuki-Miyaura coupling with small molecules. In this context, they present attractive alternatives to homogenous catalysts owing to their facile separation from final products, possible reusability and cost-effectiveness as no elaborate ligand is necessary for their stabilisation. ${ }^{[13]}$ However, most reported Pd NPs-mediated reactions require the use of organic co-solvents, surfactants, strong bases and/or high temperatures, which are not compatible with biological applications. ${ }^{[14]}$ In our continued effort to develop efficient nanotechnologies to promote biologically compatible reactions, we report here the elaboration of palladium nanoparticles (ca. $5.6 \mathrm{~nm}$ diameter) stabilized on the surface of larger poly(D,L-lactide-co-glycolide)-blockpoly(ethylene glycol) copolymer nanoparticles (PLGA-PEG NPs, ca. $158 \mathrm{~nm}$ diameter) as catalysts for the SuzukiMiyaura cross-coupling reaction between halogenated amino acids and aryl-boron reagents under physiologically-relevant conditions. The prepared palladium-PLGA-PEG (Pd-PLGA-PEG) nanoassemblies (176 $\mathrm{nm}$ mean diameter) were found to be good catalysts for the coupling of brominated or iodinated amino acids with aryl boronic acids in phosphate buffer. Interestingly, the coupling with a stable water-soluble cyclic triolborate was even more efficient, yielding up to $98 \%$ conversion in $2 \mathrm{~h}$ at $37^{\circ} \mathrm{C}$ in the presence of only $1 \mathrm{~mol} \%$ of palladium, even at slightly acidic $\mathrm{pH}$. The remarkable activity of these palladium-loaded nanoassemblies even in the absence of added base, the stability and the water-solubility of the cyclic triolborate ${ }^{[15]}$ make these reaction conditions a potential tool for the modification of biological systems through carbon-carbon bond formation.

\section{Results and Discussion}

\section{Preparation of Palladium-loaded Nanoassemblies}

The palladium-loaded PLGA-PEG nanoparticles were assembled according to a straightforward two-step process. Stable PLGA-PEG nanoparticles $(157.9 \mathrm{~nm} \pm 7.8$ $\mathrm{nm}$ hydrodynamic diameter, as revealed by dynamic light scattering (DLS) analysis) were prepared according to the emulsion-evaporation method. These polymeric nanoparticles were then incubated with an aqueous solution of $\mathrm{K}_{2} \mathrm{PdCl}_{4}$. The mixture was stirred vigorously for $15 \mathrm{~h}$ at $25^{\circ} \mathrm{C}$. Excess palladium was then removed from the stable palladium-loaded nanoassemblies by ultracentrifugation. Cryogenic transmission electron microscopy (cryoTEM) images of the Pd-PLGA-PEG nanoassemblies (Fig. 1A and B) showed palladium nanoparticles of $5.6 \pm 1.2 \mathrm{~nm}$ diam- eter (Fig. 1D) positioned on the surface of the polymeric nanoparticles. Inductivelycoupled plasma mass spectrometry (ICPMS) quantified the presence of 4.8 atoms of palladium/polymer chain $(\mathrm{Pd} /$ polymer mass ratio $=0.01)$. DLS analysis revealed that palladium loading resulted in an increase in diameter going from $157.9 \pm 7.8$ $\mathrm{nm}$ for PLGA-PEG NPs to $176.3 \pm 6.8$ $\mathrm{nm}$ for Pd-PLGA-PEG nanoassemblies. Reduction of the negative surface charge (Zeta potential) from $-14.32 \pm 0.45 \mathrm{mV}$ to $-7.68 \pm 5.71 \mathrm{mV}$ was also observed upon palladium loading (Table 1).

Despite their low absolute surface charge, the Pd-PLGA-PEG nanoassemblies showed reasonable stability, with no significant aggregation at least 3 days after

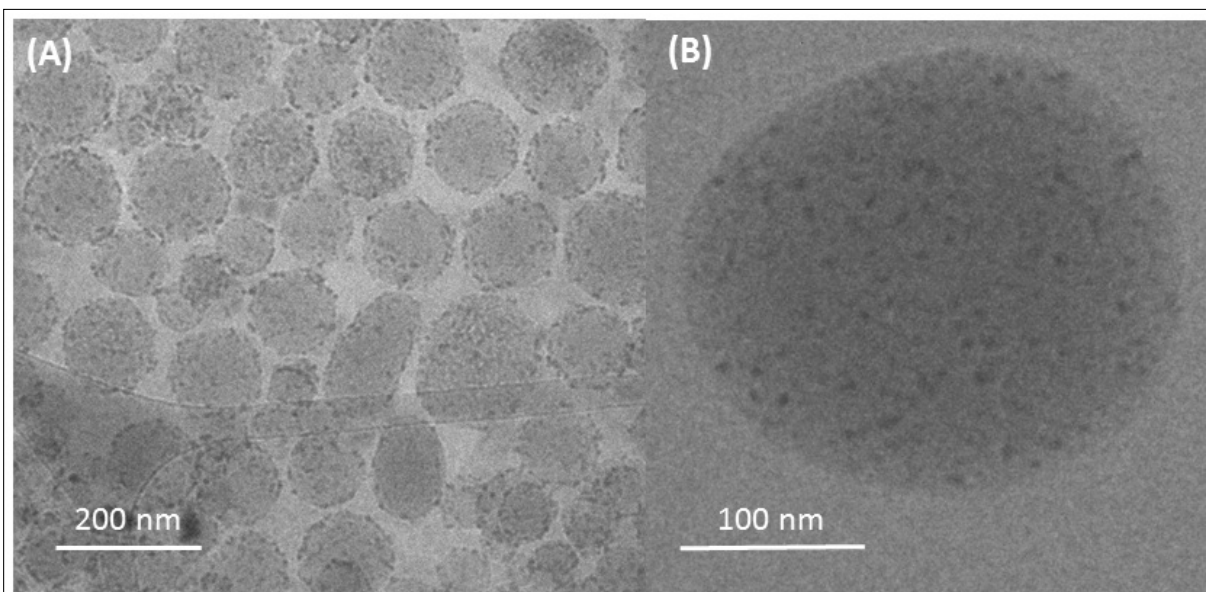

(c)

Stability of Pd-PLGA-PEG

(D)
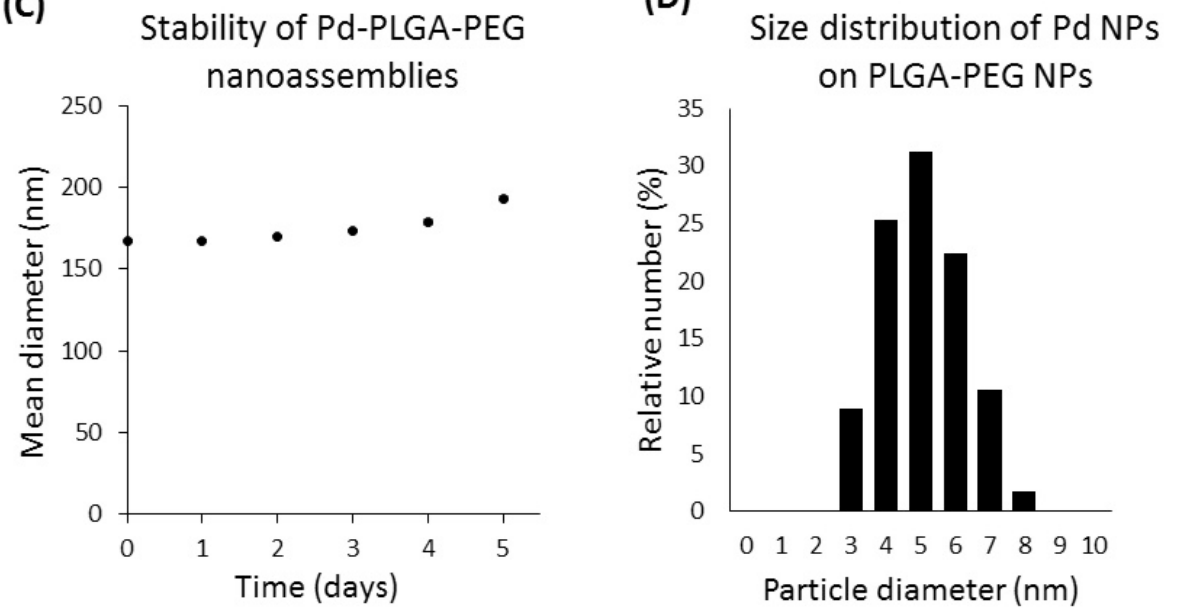

Fig. 1. Cryo-TEM images of $(A)$ a representative sample and $(B)$ a zoom of Pd-PLGA-PEG nanoassemblies. (C) Evolution of the mean diameter of Pd-PLGA-PEG nanoassemblies upon incubation at $25^{\circ} \mathrm{C}$ in water for several days after resuspension (PLGA-PEG concentration = $0.2 \mathrm{mg}$ / $\mathrm{mL}$ ), (D) Size distribution of the palladium nanoparticles on the PLGA-PEG (304 randomly chosen samples on cryo-TEM images, mean diameter: $5.59 \mathrm{~nm} \pm 1.17 \mathrm{~nm}$ )

Table 1. Physicochemical characterisation of PLGA-PEG NPs and Pd-PLGA-PEG nanoassemblies (NAs). Each value represents the average of more than six experiments \pm standard deviation.

\begin{tabular}{|c|c|c|c|}
\hline & $\begin{array}{c}\text { Hydrodynamic } \\
\text { diameter [nm] }\end{array}$ & $\begin{array}{c}\text { Polydispersity } \\
\text { index }\end{array}$ & $\begin{array}{c}\text { Zeta potential } \\
{[\mathbf{m V}]}\end{array}$ \\
\hline PLGA-PEG NPs & $157.9 \pm 7.8$ & $0.094 \pm 0.016$ & $-14.32 \pm 0.45$ \\
\hline Pd-PLGA-PEG NAs & $176.3 \pm 6.8$ & $0.113 \pm 0.039$ & $-7.68 \pm 5.71$ \\
\hline
\end{tabular}


tively as determined by ${ }^{1} \mathrm{H}-\mathrm{NMR}$ (Table 2 , entry 1). Surprisingly, reaction with the 4-bromo-analog (2) showed $43 \%$ and $98 \%$ conversion efficiency after 2 and $18 \mathrm{~h}$ respectively under the same conditions (Table 2, entry 2). Although aryl bromides are usually less reactive than their iodinated analogues in the Suzuki-Miyaura reaction, ${ }^{[16]}$ the poor solubility of $\mathrm{N}$-Boc-4iodo-L-phenylalanine in phosphate buffer might explain the reversed reactivity pattern observed here. However, coupling of the chlorinated analogue (3) did not give the desired product under the mild conditions used here (Table 2, entry 3 ). When applied to $N$-Boc-3-iodo-L-tyrosine (4), our standard conditions yielded the expected coupling product in $67 \%$ and $70 \%$ conversion after 2 and $18 \mathrm{~h}$ respectively (Table 2, entry 4).

Exploring the electronic effect of substituents on the phenyl boronic acid revealed that, as expected, substitution on the 3-position of the phenyl ring with an electron-withdrawing nitro group, significantly slowed down the reaction rate, probably due to a decreased nucleophilicity of the boron reagent, disfavouring the transmetallation step. In this case, no reaction was observed with $N$-Boc-4-bromoL-phenylalanine (2) whereas, the coupling with $N$-Boc-4-iodo-L-phenylalanine (1) afforded the corresponding adduct in $35 \%$ conversion in $2 \mathrm{~h}$ (Table 2, entry 5). By contrast, an electron-donating methoxy group on the 4-position of the phenyl boronic acid facilitated the reaction. Thus, as expected from an increased nucleophilicity of the boron coupling partner, coupling of $N$-Boc-4-bromo-L-phenylalanine (2) with 4-methoxyphenylboronic acids was found more efficient than in the absence of substituent, giving $62 \%$ conversion in $2 \mathrm{~h}$ (Table 2, entry 6).

Aryl boronic acids are the most widely used coupling partners in the SuzukiMiyaura cross-coupling reaction, nevertheless they can suffer from a poor stability and give side reactions such as protodeboronation, di- and trimerization leading to the formation of byproducts, reducing yields and demanding laborious purification processes. ${ }^{[17]}$ Alternative organoboron reagents, such as pinacol esters, potassium trifluoroborates, or boronates, display improved physico-chemical properties providing clear advantages over standard boronic acids under specific Suzuki-Miyaura coupling conditions. To investigate the effect of the boron species on the reaction outcome, different water-soluble boronic coupling partners were tested in a model system involving the halogenated $N$-BocL-phenylalanine derivatives $\mathbf{1 - 3}$ with phenyl-boron reagents 5-7 in the presence of $1 \mathrm{~mol} \%$ of PLGA-PEG-supported palladium. Aryltrifluoroborate salts and

Table 2. Suzuki-Miyaura cross-coupling reactions between halogenated amino acid and phenyl boronic acid derivatives catalyzed by Pd-PLGA-PEG nanoassemblies.

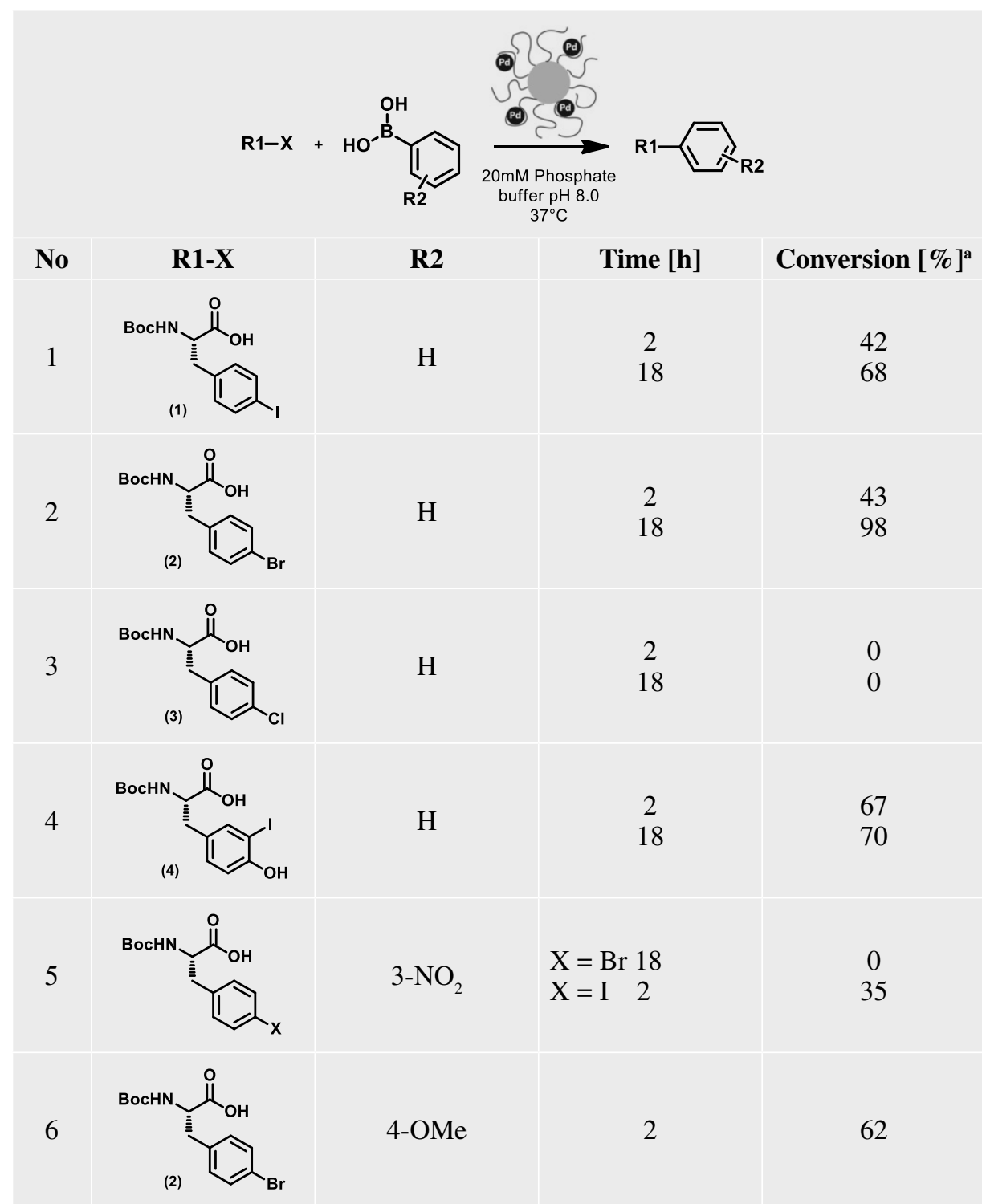

1 equiv. R1-X, 3 equiv. R-B $(\mathrm{OH})_{2}$, 1 mol\% Pd on PLGA-PEG nanoparticles. ${ }^{\text {aT }}$ The conversion was determined by ${ }^{1} \mathrm{H}-\mathrm{NMR}$ spectroscopy on the crude reaction mixture.

6-methyl-2-phenyl-1,3,6,2-dioxazaborocane-4,8-dione (phenyl boronic MIDA esters) are known partners of the SuzukiMiyaura cross-coupling reaction with the advantage of being monomeric, crystalline and air- and moisture-stable reagents. ${ }^{[18]}$ Although usually more efficient than the corresponding boronic acids in some reaction settings, ${ }^{[15 a]}$ little or no reaction was observed with our system after $18 \mathrm{~h}$ (Table 3 , entry $1 \& 2$ ).

The air- and moisture-stable tetracoordinated boronate complex 7 was then tested. These reagents were reported as useful reaction partners in Suzuki-Miyaura cross-couplings as the quaternization of the boron centre with the anionic ligand enhanced significantly the nucleophilicity of the organic groups attached, facilitating transmetallation on the metal centre. ${ }^{[15]}$ As a result, such reagents have been reported to react in the Suzuki-Miyaura cross-coupling reaction even in the absence of added base. The phenyl cyclic triolborate derivative (7) was prepared from phenyl boronic acid following a reported procedure. ${ }^{[15 b]}$ Interestingly, the reaction of this compound with $N$-Boc-4-iodo-L-phenylalanine (1) in the presence of the catalytic Pd-PLGAPEG nanoassemblies led to excellent conversion in $2 \mathrm{~h}$ in $\mathrm{pH} 8.0$ phosphate buffer at $37{ }^{\circ} \mathrm{C}$ (Table 3 , entry 3 ). The reaction with the less reactive 4-bromo-phenylalanine analogue (2) led to $90 \%$ and $98 \%$ conversion in 2 and $18 \mathrm{~h}$ respectively (Table 3 , entry 4). However, by analogy with the observation made with the arylboronic acids, no reaction was observed with $\mathrm{N}$-Boc-4chloro-L-phenylalanine (3) (Table 3, entry 5). Remarkably, although Suzuki-Miyaura cross-couplings with aryl boronic acids usually require the addition of an exoge- 
Table 3. Suzuki-Miyaura cross-coupling reactions between halogenated phenylalanine and various organoboron reagents catalysed by Pd-PLGA-PEG nanoassemblies
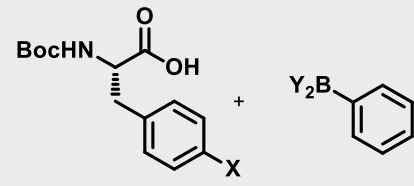

No. $\mathbf{X}$

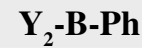<smiles>[Pb]c1ccccc1</smiles>

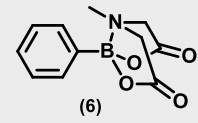

pH

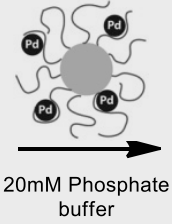<smiles>O=C(CNC(=O)[C@H](Cc1ccccc1)C(=O)O)OCc1ccccc1</smiles>
buffer
$37^{\circ} \mathrm{C}$

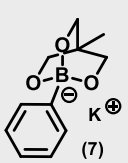

8.0

2

18

98

3 I

(7)

$4 \mathrm{Br}$

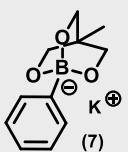

8.0

18

2

97

6.0

97

1 equiv. R1-X, 3 equiv. $Y_{2} B-P h, 1$ mol\% Pd on PLGA-PEG nanoparticles. ${ }^{\text {TT }}$ The conversion was determined by ${ }^{1} \mathrm{H}-\mathrm{NMR}$ spectroscopy on the crude reaction mixture.

nous base, the reaction between $N$-Boc4-iodo-L-phenylalanine (1) and the triolborate (7) catalysed by the palladium-assemblies gave excellent conversions in $2 \mathrm{~h}$ even in neutral and slightly acidic buffers (97\% conversion at $\mathrm{pH} 7.0$ and 6.0) (Table 3 , entries $6 \& 7$ ). The remarkable activity of these palladium-loaded nanoassemblies in the absence of added bases represents a significant advantage over standard Suzuki-Miyaura coupling conditions for reactions involving base-sensitive reaction partners or where little control over the $\mathrm{pH}$ is possible.

Interestingly, the process is not limited to the micromolar scale but could be scaled to afford milligram quantities. The reaction of $33 \mathrm{mg}$ of $N$-Boc-4-iodo-Lphenylalanine (1) with 7 in the presence of
Pd-PLGA-PEG nanoassemblies ( $1 \mathrm{~mol} \%$ palladium) showed complete conversion after $2 \mathrm{~h}$ at $37^{\circ} \mathrm{C}$ in phosphate buffer. The coupled product was isolated by column chromatography in $81 \%$ yield validating the usefulness of our process for synthetic transformations of polyfunctional, water-soluble substrates under mild conditions.

\section{Conclusion and Perspectives}

The Pd-PLGA-PEG nanoassemblies reported here showed excellent catalytic activities for the coupling of halogenated amino acid analogues via Suzuki-Miyaura reaction in water and mild conditions without the need for additional co-solvents or surfactants. Noteworthy is the remarkable efficiency of the coupling process when using cyclic triolborate reagents as boron partners. Indeed, the reaction of $N$-Boc4-iodo-L-phenylalanine (1) with a phenyl cyclic triolborate derivative 7 led to $98 \%$ conversion in $2 \mathrm{~h}$ in the presence of $1 \mathrm{~mol} \%$ palladium in aqueous phosphate buffer at $37^{\circ} \mathrm{C}$, near-physiological $\mathrm{pH}$ and on a synthetically relevant scale.

The Suzuki-Miyaura cross-coupling variant reported here works even at slightly acidic $\mathrm{pH}$. This will present a significant advantage compared to the original version of the reaction in environments where the $\mathrm{pH}$ cannot be controlled, such as in the intracellular space. In addition, the chelating effect of the polymer chains stabilising the metal may reduce non-specific interactions with surrounding biomolecules, and thus minimize toxicity as compared to small-molecule-stabilized homogenous catalysts. In this context, we believe that the present approach will contribute to the design of a catalytic system able to selectively achieve an irreversible carbon-carbon bond formation in complex biological systems.

\section{Material and Methods}

\section{Materials}

Unless otherwise stated, all reagents and solvents were purchased from SigmaAldrich. Potassium tetrachloropalladate (II) $\left(\mathrm{K}_{2} \mathrm{PdCl}_{4}\right) 99.99 \%$ trace metal basis (Sigma-Aldrich, Lot $\mathrm{N}^{\circ}$ MKBL2083V) was used as a palladium source.

$N$-Boc-4-iodo-L-phenylalanine, $\mathrm{N}$-Boc-4-bromo-L-phenylalanine and $\mathrm{N}$-Boc-4-chloro-L-phenylalanine were purchased from Alfa Aesar. $N$-Boc-3-iodoL-tyrosine was prepared as reported elsewhere. ${ }^{[19]}$ PLGA-PEG (poly(ethylene glycol)-poly(DL-lactide-co-glycolide) 50:50 Resomer $^{\circledR}$ PEG type RGP d 50105 (MW $50000 \mathrm{~g} / \mathrm{mol}$ ) (diblock, 10\% PEG with 5000 Dalton, Evonik Industries AG, Essen, Germany) was generously donated by the group of Prof. Elias Fattal, Université Paris-Saclay. Deionized MilliQ purified water was used for all chemical reactions and for nanoparticles synthesis. Proton nuclear magnetic resonance $\left({ }^{1} \mathrm{H}\right.$ NMR) spectra were recorded on a Bruker AV500 (500 $\mathrm{MHz}$ ) or Bruker AV400 (400 MHz). All chemical shifts are quoted on the $\delta$ scale in ppm using residual solvent as the internal standard. Ultracentrifugation was achieved using Optima LE-80K Ultracentrifuge Beckman Coulter apparatus.

\section{PLGA-PEG Nanoparticle (PLGA- PEG NPs) Preparation}

Plain PLGA-PEG nanoparticles were prepared by emulsion-evaporation. 
Practically, PLGA-PEG (50 mg) was dissolved into $\mathrm{CH}_{2} \mathrm{Cl}_{2}$ (4 mL). The organic phase was emulsified into $10 \mathrm{~mL}$ of $1.5 \%$ sodium cholate (w/v) aqueous solution using a vortex for $1 \mathrm{~min}$ and then a vibrating metallic tip at $180 \mathrm{~V}$, at $30 \%$ amplitude for $1 \mathrm{~min}$ at $0{ }^{\circ} \mathrm{C}$. The organic solvent was evaporated by magnetic stirring for $4 \mathrm{~h}$. The nanoparticles were recovered by ultracentrifugation $\left(1 \mathrm{~h}, 27440 \mathrm{~g}, 4{ }^{\circ} \mathrm{C}\right)$ and the pellet was resuspended in MilliQ water to form a stock suspension of $5 \mathrm{mg}$ PLGA$\mathrm{PEG} / \mathrm{mL}$.

\section{Pd-loading on PLGA-PEG Nanoparticles (Pd-PLGA-PEG NPs)}

PLGA-PEG NPs stock suspension (200 $\mu \mathrm{L}, 1 \mathrm{mg}$ PLGA-PEG, $0.02 \mu \mathrm{mol}$ ) was diluted with $990 \mu \mathrm{L}$ MilliQ water. $\mathrm{K}_{2} \mathrm{PdCl}_{4}$ stock solution $(10 \mu \mathrm{L}, 100 \mathrm{mM}, 1 \mu \mathrm{mol})$ was added and the mixture was briefly vortexed and then incubated on a thermostated shaker (bioSan TS-100) at $25{ }^{\circ} \mathrm{C}$ and 800 $\mathrm{rpm}$ for $15 \mathrm{~h}$. The resulting nanoassemblies were recovered by ultracentrifugation $(1 \mathrm{~h}$, $24696 \mathrm{~g}, 4{ }^{\circ} \mathrm{C}$ ), the supernatant was removed and the pellet was resuspended in $450 \mu \mathrm{L}$ MilliQ water.

\section{Size and Zeta Potential}

The hydrodynamic diameter $(\mathrm{dH})$ and polydispersity index (PDI) of the PLGAPEG NPs and Pd-PLGA-PEG nanoassemblies were measured by quasi elastic light scattering, using a Zetasizer Nano ZS instrument (Malvern, France). Suspensions were diluted in water and filtered over a $1 \mu \mathrm{m}$-pore glass filter. Measurements were performed in triplicate at $20^{\circ} \mathrm{C}$, at an angle of $173^{\circ}$ to avoid multiple scattering. The counting time was set at $60 \mathrm{~s}$. Zeta potential measurements were carried out with the same instrument, at $25^{\circ} \mathrm{C}$, in $1 \mathrm{mM}$ $\mathrm{NaCl}$. The average palladium nanoparticle size and size distribution on the surface of the PLGA-PEG NPs were obtained from randomly chosen particles on Cryo-TEM images using imageJ software.

\section{Cryogenic Transmission Electron Microscopy (cryo-TEM)}

For cryogenic transmission electron microscopy (cryo-TEM) analysis, $5 \mu \mathrm{L}$ of nanoassembly suspension at $5 \mathrm{mg} / \mathrm{mL}$ were deposited onto a Lacey Formvar/carbon 300 Mesh Copper grid (Ted Pella). Most of the drop was removed with a blotting filter paper and the residual thin film remaining was vitrified by plunging into a bath of liquid ethane slush. The grids were stored under liquid nitrogen temperature until transfer to a 626 Single Tilt Cryotransfer System (Gatan Inc.) and observed with a JEOL $2100 \mathrm{HC}$ microscope operated at 200KV (JEOL Europe SAS). Images were recorded under low dose conditions on a Gatan Ultrascan 4k x 4k Digital (CCD)
Camera System Camera at a nominal magnification of $80 \mathrm{x}$ at a defocus level of $2 \mu \mathrm{m}$.

\section{Determination of Pd Encapsulation Efficacy by ICP-MS}

The association of Pd to PLGA-PEG nanoparticles (i.e. encapsulation efficacy) was determined by inductively coupled plasma-mass spectrometry (ICP-MS) by Antellis (Parc technologique du Canal, 3 rue des satellites, 31400 Toulouse).

\section{Potassium 4-methyl-1-phenyl- 2,6,7-trioxa-1-borate-bicyclo[2.2.2] octane (7)}

A mixture of phenylboronic acid (1 mmol, $121.9 \mathrm{mg}, 1$ equiv.) and 1,1,1-tris(hydroxymethyl)ethane ( $1 \mathrm{mmol}$, $120.1 \mathrm{mg}, 1$ equiv.) in toluene ( $2 \mathrm{~mL}$ ) was heated under reflux with azeotropic distillation using a Dean-Stark apparatus for 4 h. The solvent was removed under reduced pressure and the residue taken up into toluene ( $2 \mathrm{~mL})$ and solid $\mathrm{KOH}$ (0.9 mmol, 50.4 $\mathrm{mg}, 0.9$ equiv.) was added. The reaction mixture was heated at reflux with removal of the water by the Dean-Stark method for $4 \mathrm{~h}$. The solid that precipitated was filtered, washed with acetone and dried under reduced pressure to afford the expected salt 7 (219.6 mg, 90\% yield). ${ }^{1} \mathrm{H}$ NMR (300 $\mathrm{MHz}, \mathrm{DMSO}) \delta 7.30(\mathrm{~d}, J=7.2 \mathrm{~Hz}, 2 \mathrm{H})$, $6.92(\mathrm{~m}, 3 \mathrm{H}), 3.56(\mathrm{~s}, 6 \mathrm{H}), 0.47(\mathrm{~s}, 3 \mathrm{H}) .{ }^{13} \mathrm{C}$ NMR (75 MHz, DMSO) $\delta 132.61,125.86$, 124.31, 74.19, 16.76, (C-B signal was not detected).

\section{General Method for Suzuki- Miyaura Coupling on Small Scale}

In an Eppendorf tube, the arylhalide ( $0.008 \mathrm{mmol}, 1$ equiv.) and the boronic acid $(0.024 \mathrm{mmol}, 3$ equiv.) were suspended in phosphate buffer pH 8.0 (200 mM, 50 $\mu \mathrm{L})$. The suspension of Pd-PLGA-PEG nanoassemblies $(0.00008 \mathrm{mmol} \mathrm{Pd}, 0.01$ equiv., $450 \mu \mathrm{L}$ ) was added and the reaction was mixed and shaken on a thermostated shaker (bioSan TS-100) at 800rpm at $37^{\circ} \mathrm{C}$. After $2 / 18 \mathrm{~h}$, the reaction was frozen in liquid nitrogen and the solution lyophilised. The crude product was dissolved in MeOD and analysed by ${ }^{1} \mathrm{H}-\mathrm{NMR}$.

\section{Synthesis of N-Boc-4-phenyl-L- phenylalanine via Suzuki-Miyaura coupling involving cyclic triol- borate 7}

$\mathrm{N}$-Boc-4-iodo-L-phenylalanine $\quad(0.08$ mmol, $31.3 \mathrm{mg}, 1$ equiv.) and potassium 4-methyl-1-phenyl-2,6,7-trioxa-1-borate-bicyclo[2.2.2] octane (7) $(0.240 \mathrm{mmol}$, $58.5 \mathrm{mg}, 3$ equiv.) were dissolved in phosphate buffer $(200 \mathrm{mM}, 500 \mu \mathrm{L})$. Then Pd-PLGA-PEG nanoassemblies $(0.0008$ $\mathrm{mmol} \mathrm{Pd}, 0.01$ equiv., $4500 \mu \mathrm{L}$ ) were added and the reaction was stirred at $37{ }^{\circ} \mathrm{C}$. After $2 \mathrm{~h}$, the solution was acidified with a citric acid solution $10 \%(5 \mathrm{~mL})$. The aqueous solution was extracted with EtOAc ( $5 \mathrm{x}$ $5 \mathrm{~mL}$ ). The combined organic layers were then washed with brine $(10 \mathrm{~mL})$, dried over $\mathrm{MgSO}_{4}$ filtered and concentrated. The residue was purified by chromatography on silica gel column (100/0 to $95 / 5$ DCM/ $\mathrm{MeOH}$ ) to provide the expected $N$-Boc-4phenyl-L-phenylalanine (22.1 $\mathrm{mg}, 81 \%$ ). ${ }^{1} \mathrm{H}$ NMR (500 MHz, MeOD) $\delta 7.47$ (d, $J=$ $7.3 \mathrm{~Hz}, 2 \mathrm{H}), 7.40$ (d, $J=7.7 \mathrm{~Hz}, 2 \mathrm{H}), 7.30$ $(\mathrm{t}, J=7.6 \mathrm{~Hz}, 2 \mathrm{H}), 7.20(\mathrm{~d}, J=7.4 \mathrm{~Hz}, 3 \mathrm{H})$, $4.21(\mathrm{~m}, 1 \mathrm{H}), 3.11(\mathrm{dd}, J=13.6,4.8 \mathrm{~Hz}$, 1H), 2.86 (dd, $J=13.6,8.0 \mathrm{~Hz}, 1 \mathrm{H}), 1.24$ (s, 9H). MS (ESI+) m/z (\%): 364.1 (100\%) $[\mathrm{M}+\mathrm{Na}]^{+}$.

\section{Acknowledgements}

This work is supported by a public grant overseen by the French National Research Agency (ANR) as part of the 'Investissements d'Avenir' program (Labex NanoSaclay, reference: ANR-10-LABX-0035). We thank Jean-François Gallard (Institut de Chimie des Substances Naturelles, CNRS-ICSN UPR 2301, Université Paris-Sud) and Camille Dejean (BioCIS, UMR-CNRS-8076, Université Paris-Sud) for technical assistance on NMR measurements; The Service d'Analyse des Médicaments et Métabolites (SAMM) and Stéphanie Nicolaÿ for technical assistance on mass spectrometry measurements; Ghislaine Frébourg (Service de Microscopie Electronique de l'IFR de Biologie Intégrative, la FRM 2006, SESAME 2005 et le CNRSINSB) and Julie Mougin (Institut Galien, UMR CNRS 8612, Université Paris-Sud) for technical assistance on cryo-TEM measurements. AD thanks the Swiss National Science Foundation for funding.

Received: January 15, 2016

[1] a) J. A. Prescher, C. R. Bertozzi, Nat. Chem Biol. 2005, 1, 13; b) M. Grammel, H. C. Hang, Nat. Chem. Biol. 2013, 9, 475

[2] a) E. M. Sletten, C. R. Bertozzi, Angew. Chem. Int. Ed. 2009, 48, 6974; b) K. Lang, J. W. Chin, Acs Chem. Biol. 2014, 9, 16.

[3] M. King, A. Wagner, Bioconjugate Chem. 2014, 25,825 .

[4] a) E. M. Sletten, C. R. Bertozzi, Acc. Chem. Res. 2011, 44, 666; b) A. C. Knall, C. Slugove, Chem. Soc. Rev. 2013, 42, 5131 .

[5] a) L. I. Willems, W. A. Van der Linden, N. Li, K. Y. Li, N. Liu, S. Hoogendoorn, G. A. Van der Marel, B. I. Florea, H. S. Overkleeft, Acc. Chem. Res. 2011, 44, 718; b) Z. Q. Li, D. Y. Wang, L. Li, S. J. Pan, Z. K. Na, C. Y. J. Tan, S. Q. Yao, J. Am. Chem. Soc. 2014, 136, 9990; c) J. Li, S. Jia, P. R. Chen, Nat. Chem. Biol. 2014, 10, 1003 .

[6] a) R. Ekkebus, S. I. van Kasteren, Y. Kulathu, A. Scholten, I. Berlin, P. P. Geurink, A. de Jong, S. Goerdayal, J. Neefjes, A. J. R. Heck, D. Komander, H. Ovaa, J. Am. Chem. Soc. 2013, 135, 2867; b) M. F. Debets, S. S. Van Berkel, J. Dommerholt, A. J. Dirks, F. P. J. T. Rutjes, F. L. Van Delft, Acc. Chem. Res. 2011, 44, 805; c) M. T. Taylor, M. L. Blackman, O. Dmitrenko, J. M. Fox, J. Am. Chem. Soc. 2011, 133, 9646; d) J. Li, P. R. Chen, ChemBioChem 2012, 13, 1728; e) H. E. Murrey, J. C. Judkins, C. W. A. Ende, T. E. Ballard, Y. Z. Fang, K. Riccardi, L. Di, E. 
R. Guilmette, J. W. Schwartz, J. M. Fox, D. S. Johnson, J. Am. Chem. Soc. 2015, 137, 11461.

[7] H. W. Shih, D. N. Kamber, J. A. Prescher, Curr. Opin. Chem. Biol. 2014, 21, 103.

[8] S. V. Chankeshwara, E. Indrigo, M. Bradley, Curr. Opin. Chem. Biol. 2014, 21, 128.

[9] a) R. M. Yusop, A. Unciti-Broceta, E. M. V. Johansson, R. M. Sanchez-Martin, M. Bradley, Nat. Chem. 2011, 3, 239; b) K. Kodama, S. Fukuzawa, H. Nakayama, K. Sakamoto, T. Kigawa, T. Yabuki, N. Matsuda, M. Shirouzu, K. Takio, S. Yokoyama, K. Tachibana, ChemBioChem 2007, 8, 232

[10] J. M. Chalker, C. S. C. Wood, B. G. Davis, J. Am. Chem. Soc. 2009, 131, 16346.

[11] C. D. Spicer, B. G. Davis, Chem. Commun. 2011, 47, 1698.
[12] N. Li, R. K. V. Lim, S. Edwardraja, Q. Lin, J. Am. Chem. Soc. 2011, 133, 15316.

[13] a) M. Pérez-Lorenzo, J. Phys. Chem. Lett. 2012, 3, 167; b) P. Taladriz-Blanco, P. Herves, J. Perez-Juste, Top. Catal. 2013, 56, 1154

[14] a) B. J. Gallon, R. W. Kojima, R. B. Kaner, P. L. Diaconescu, Angew. Chem. Int. Ed. 2007 46, 7251; b) S. W. Kim, M. Kim, W. Y. Lee, T. Hyeon, J. Am. Chem. Soc. 2002, 124, 7642; c) Y. Li, X. M. Hong, D. M. Collard, M. A. ElSayed, Org. Lett. 2000, 2, 2385; d) C. Liu, Q. J. Ni, P. P. Hu, J. S. Qiu, Org. Biomol. Chem. 2011, 9, 1054; e) Y. Z. Piao, Y. J. Jang, M Shokouhimehr, I. S. Lee, T. Hyeon, Small 2007, 3, 255; f) A. Prastaro, P. Ceci, E. Chiancone, A. Boffi, R. Cirilli, M. Colone, G. Fabrizi, A Stringaro, S. Cacchi, Green Chem. 2009, 11,
1929; g) H. Veisi, A. R. Faraji, S. Hemmati, A. Gil, Appl. Organomet. Chem. 2015, 29, 517.

[15] a) A. J. J. Lennox, G. C. Lloyd-Jones, Chem. Soc. Rev. 2014, 43, 412; b) Y. Yamamoto, M. Takizawa, X. Q. Yu, N. Miyaura, Angew. Chem. Int. Ed. 2008, 47, 928.

[16] J. K. Stille, K. S. Y. Lau, Acc. Chem. Res. 1977, $10,434$.

[17] D. G. Hall, in 'Boronic Acids', Wiley-VCH Verlag GmbH \& Co. KGaA, 2006, pp. 1-99.

[18] a) D. M. Knapp, E. P. Gillis, M. D. Burke, J. Am. Chem. Soc. 2009, 131, 6961; b) G. A. Molander, B. Canturk, Angew. Chem. Int. Ed. 2009, 48, 9240.

[19] A. Dumas, C. D. Spicer, Z. H. Gao, T. Takehana, Y. Y. A. Lin, T. Yasukohchi, B. G. Davis, Angew. Chem. Int. Ed. 2013, 52, 3916. 